CLINICAL STUDY

\title{
Elevated plasma glucose-dependent insulinotropic polypeptide associates with hyperinsulinemia in metabolic syndrome
}

\author{
Salvatore Calanna, Francesca Urbano, Salvatore Piro, Rose Maria Zagami, Antonino Di Pino, Luisa Spadaro, \\ Francesco Purrello and Agata Maria Rabuazzo \\ Department of Clinical and Molecular Biomedicine, University of Catania, Ospedale Garibaldi Nesima, Via Palermo 636, 95122 Catania, Italy \\ (Correspondence should be addressed to F Purrello; Email: fpurrell@unict.it)
}

\begin{abstract}
Background and aims: Metabolic syndrome (MS) is a high-risk condition for type 2 diabetes, a disease characterized by insulin resistance and insulin secretion abnormalities. Insulin resistance has been widely characterized in MS subjects while insulin secretion has been poorly investigated. The present study was hence undertaken to further investigate the $\alpha$ and $\beta$ cell function and entero-insular axis in this pre-diabetic condition.

Materials and methods: Using $120^{\prime}$ oral glucose tolerance test (OGTT, $75 \mathrm{~g}$ ) and $60^{\prime}$ intravenous glucose tolerance test (IVGTT, $0.3 \mathrm{~g} / \mathrm{kg}$ ), we studied $\alpha$ and $\beta$ cell function, insulin resistance, and incretin levels in 96 subjects with normal fasting glucose and normal glucose tolerance to OGTT, with (MS,$+ n=29$ ) and without MS (MS,$- n=67)$.

Results: MS + individuals showed in comparison with MS - : higher insulinogenic index (IG30) and higher area under the curve (AUC) (0-120) for glucose and insulin during the OGTT, $P<0.05$; higher AUC $(0-10)$ for glucose $(P<0.05)$ but similar first phase insulin secretion $(P=N S)$ as measured by $\triangle$ AIRG and AUC (0-10) for insulin during the IVGTT; increased AUC (0-60) for insulin during the IVGTT $(P=0.04)$; higher GIP levels at 30' $(P=0.03)$, 60 $(P=0.01), 90^{\prime}(P=0.003)$, and $120^{\prime}$ $(P=0.004)$; higher AUC $(0-120)$ for GIP $(P=0.007)$; similar AUC $(0-120)$ for GLP-1 during the OGTT; and delayed glucagon suppression after the OGTT.

Conclusion: NGT subjects with MS showed increased GIP secretion that could be responsible for the delayed glucagon suppression during the OGTT, thereby suggesting a role for incretins in regulating glucose homeostasis in this condition.
\end{abstract}

European Journal of Endocrinology 166 917-922

\section{Introduction}

Obesity has become a serious worldwide healthcare problem, reaching in the last decades pandemic proportions. In the general population, the overall relative risk of diabetes for obese people compared with those with normal weight is 7.19 , and for overweight people it is 2.99 (1). Current evidence suggests that, among obese and overweight subjects, metabolic syndrome (MS) confers a further higher risk of type 2 diabetes mellitus by reducing glucose sensitivity. In particular, insulin resistance as a consequence of excess central adiposity has been thought to be a key event in the progression of MS.

However, insulin resistance alone rarely causes diabetes, since glucose homeostasis depends on the balance between insulin sensitivity and insulin secretion (2). Whereas insulin resistance has been extensively characterized in subjects with MS, only a few studies have examined insulin secretion in these subjects.
The present study was hence undertaken to investigate the pathways through which MS influences insulin secretion. To investigate whether MS exerts a direct effect on the $\beta$ cell function, we studied the first phase of insulin secretion in response to an oral glucose tolerance test (OGTT) load (75 g) and to an intravenous glucose tolerance test (IVGTT, $0.3 \mathrm{~g} / \mathrm{kg}$ ) in normoglucose-tolerant subjects with and without MS.

Incretin response to oral nutrients and $\alpha$ cell function have also been poorly investigated in these patients. The incretin effect accounts for $50-70 \%$ of insulin secretion after oral glucose load (3), and $80 \%$ of the incretin effect on insulin secretion is attributed to only two hormones: GLP-1 and GIP. As far as glucagon is concerned, GLP-1 has been shown to be glucagonostatic (4), whereas GIP has been shown to enhance glucagon secretion (5).

To investigate the entero-insular hormones, we evaluated incretin secretion during the OGTT (75 g). Finally, we measured both basal glucagon levels and their response to the OGTT. 


\section{Research design and methods}

\section{Participants}

We recruited a total of 139 subjects for this study. They were consecutively enrolled among patients attending our university hospital for cardiovascular risk evaluation. Inclusion criteria were: body mass index (BMI) $\geq 25 \mathrm{~kg} / \mathrm{m}^{2}$, fasting plasma glucose $\leq 100 \mathrm{mg} / \mathrm{dl}$, and at least one or more components of the MS. Exclusion criteria were: previous history of diabetes, use of medications known to affect glucose metabolism, previous cardiovascular events, clinical evidence of advanced liver or renal disease, and/or a recent history of acute illness. The Local Ethics Committee approved the study, and after the nature of the study had been explained to each participant, informed written consent was obtained.

\section{Experimental protocols}

All 139 participants underwent standard blood testing after a 12-h overnight fast (plasma glucose, insulin, and lipids), anthropometric measurements, and blood pressure monitoring. Subjects were divided into two groups, with or without the MS (MS+ and MS-), according to the NCEP-ATPIII criteria (6). All subjects underwent a $75 \mathrm{~g}$ OGTT: samples for glucose, insulin, glucagon, GLP-1, and GIP measurements were drawn at baseline and every $30 \mathrm{~min}$ after glucose ingestion up to and including $120 \mathrm{~min}$ and distributed into chilled tubes containing EDTA, with the addition of aprotinin (500 kIU/ml blood), for the analysis of plasma glucagon, GLP-1, and plasma GIP. For the analysis of glucose, insulin, and C-peptide, blood was distributed into chilled tubes containing heparin. All tubes were immediately cooled on ice and centrifuged at $4^{\circ} \mathrm{C}$ for $20 \mathrm{~min}$. Plasma was stored at $-20{ }^{\circ} \mathrm{C}$ until analysis. The impaired glucose tolerant and diabetic patients, according to the American Diabetes Association criteria (7) based on their 2-h post-OGTT glucose levels, were excluded from this study. Then, 29 normoglucose-tolerant MS + and 67 normoglucose-tolerant MS - subjects were included in our study. After One week after the OGTT, these patients underwent a $60^{\prime}$ IVGTT: at $-1 \mathrm{~min}$ a bolus of $0.3 \mathrm{~g} / \mathrm{kg}$ glucose was given; blood samples for glucose, insulin, and C-peptide measurements were drawn at baseline, $2^{\prime}$, $3^{\prime}, 4^{\prime}, 5^{\prime}, 10^{\prime}, 15^{\prime}, 20^{\prime}, 25^{\prime}, 30^{\prime}, 40^{\prime}, 50^{\prime}$, and $60^{\prime}$. Blood for glucose and insulin was sampled as for the OGTT.

\section{Analytical procedures}

Body weight and height were measured, and BMI was calculated as weight $(\mathrm{kg}) /$ height $\left(\mathrm{m}^{2}\right)$. Waist circumference was measured in a standing position at the level of the umbilicus. Blood pressure was measured with a calibrated mercury sphygmomanometer when the subjects had rested in the supine position for $10 \mathrm{~min}$. Plasma glucose was measured by the glucose oxidase method. Serum insulin was measured using a microparticle enzyme immunoassay (Axsym System, Abbott Laboratories). C-peptide was measured using an ELISA kit (Millipore Corporation, Billerica, MA, USA; coefficient of variation $(\mathrm{CV})$ : inter-assay, $5.0-8.7 \%$; intraassay, 1.6-4\%. Plasma glucagon was measured by a RIA (Millipore Corporation; accuracy, $97 \pm 0.8 \%$; precision $\mathrm{CV}$ : inter-assay, $11.7 \pm 3.0 \%$; intra-assay, $4.9 \pm 1.3$; sensitivity, the lowest level of human glucagon that can be detected by the assay is $18.453 \mathrm{ng} / \mathrm{l}+2$ s.D. when using a $100 \mu \mathrm{l}$ sample size; specificity: glucagon $100 \%$, oxyntomodulin $<0.1 \%$, human insulin nondetectable (ND), human C-peptide ND, somatostatin ND, and pancreatic polypeptide ND). GLP-1 (active) was measured using an ELISA kit (Millipore Corporation; accuracy, $86.9 \pm 5.2 \%$; precision $\mathrm{CV}$ : inter-assay, $8 \pm 4.8 \%$; intra-assay, $7.4 \pm 1.1$; sensitivity, the lowest level of GLP-1 that can be detected by this assay is $2 \mathrm{pmol} / \mathrm{l}$ (100 $\mu \mathrm{l}$ plasma size); specificity, GLP-1 (7-36), 100\%; GLP-1 (7-37), 100\%; GLP-1 (9-36), ND; GLP-2, ND; and Glucagon, ND). Total GIP was measured using an ELISA kit (Millipore Corporation; accuracy, $86.7 \pm 3 \%$; precision CV: inter-assay, $1.8-6.1 \%$, intraassay, 3.0-8.8\%; sensitivity, the lowest level of human GIP that can be detected by the assay is $1.64 \mathrm{pmol} / \mathrm{l}$ when using a $20-\mu \mathrm{l}$ sample size; specificity, the antibody pair used in this assay is specific to human GIP and does not significantly cross-react with glucagon, oxyntomodulin, GLP-1, and GLP-2. Serum total cholesterol, triglycerides, and HDL cholesterol were measured by available enzymatic methods. LDL cholesterol was calculated using the Friedewald formula.

\section{Calculation}

$\beta$ cell function was assessed by the insulinogenic index (IG30) calculated as the change of insulin (pmol/l) divided by the change of glucose $(\mathrm{mmol} / \mathrm{l})$ during the first $30 \mathrm{~min}$ of the OGTT $(\Delta \mathrm{IO}-30 / \Delta \mathrm{GO}-30)(8)$. During the IVGTT, as an index of insulin secretion, we used mean incremental concentrations in the 3-10-min interval following glucose bolus, defined as acute insulin response to glucose ( $\triangle$ AIRG).

$\Delta$ AIRG was divided by the incremental glucose peak, $\Delta \mathrm{Gp}$, to obtain the same units as the OGTT indices (8). The area under the insulin, C-peptide, glucose, GLP-1, and GIP was calculated by the trapezoidal method. Insulin resistance was estimated using homeostasis model of assessment (HOMA-IR), calculated as previously described (9).

\section{Statistical analysis}

Statistical comparison and analyses of clinical and biomedical parameters were performed using Stat View version 6.0 for Windows. Data are given as means \pm s.e.m. When necessary, numerical variables were logarithmically transformed to reduce skewness, and 
values were expressed as arithmetic means. Statistical analysis included unpaired $t$-test, $\chi^{2}$ test, ANOVA for continued variables. A $P$ value $<0.05$ was considered statistically significant.

\section{Results}

\section{Clinical parameters}

Clinical characteristics of the study participants are shown in Table 1.

There were no differences in age, blood pressure, CV, or BMI. Sex was not equally distributed in the different groups because of a higher number of women in the MS - group.

As expected, MS + subjects had higher triglycerides $(P<0.001)$ and lower HDL cholesterol $(P<0.001)$ compared with controls. Fasting plasma glucose, albeit normal, was slightly, but significantly, higher in MS+ $(P<0.001)$.

HOMA-IR was increased in MS $+(P<0.0001)$ and the difference was still maintained when age was used as a covariate (0.007).

\section{Plasma glucose and insulin response during OGTT}

As expected by our previous findings, MS + individuals had elevated plasma glucose levels at baseline $(P<0.01)$ and at $30^{\prime}(P<0.05$; Fig. 1A). Glucose area under the curve (AUC) (0-120) was significantly higher in $\mathrm{MS}+(848.3 \pm 23.5$ vs $775.67 \pm 17.17 \mathrm{mmol} / \mathrm{l} \times$ min, $P=0.01)$. Insulin plasma levels were statistically increased in MS subjects as shown in Fig. 1B. The early-phase insulin secretion was evaluated by insulinogenic index (IG30). In MS +, as previously reported (10), it was significantly and greatly increased (Table 2). Total insulin AUC (0-120) was also significantly higher in MS+ (Table 2).

Table 1 Clinical and metabolic characteristics of normoglucosetolerant subjects with and without MS. Data are mean \pm S.E.M.

\begin{tabular}{lccc}
\hline & $\begin{array}{c}\text { MS }+ \\
(n=29)\end{array}$ & $\begin{array}{c}\text { MS }- \\
(n=67)\end{array}$ & $\boldsymbol{P}$ value \\
\hline Age (years) & $45 \pm 2$ & $40 \pm 1$ & $\mathrm{NS}$ \\
BMI (kg/m $\left.{ }^{2}\right)$ & $34 \pm 1$ & $33 \pm 0.8$ & $\mathrm{NS}$ \\
Sex (F/M) & $15 / 14$ & $49 / 18$ & 0.02 \\
Fasting glucose (mmol/l) & $5.17 \pm 0.11$ & $4.78 \pm 0.06$ & 0.0004 \\
Waist circumference (cm) & $107 \pm 2$ & $103 \pm 2$ & $\mathrm{NS}$ \\
HDL cholesterol (mmol/l) & $1.03 \pm 0.03$ & $1.32 \pm 0.03$ & $<0.001$ \\
Triglycerides (mmol/l) & $2.2 \pm 0.17$ & $1.21 \pm 0.09$ & $<0.001$ \\
Systolic blood pressure (mmHg) & $128 \pm 3$ & $123 \pm 2$ & $\mathrm{NS}$ \\
Diastolic blood pressure (mmHg) & $79 \pm 2$ & $76 \pm 1$ & $\mathrm{NS}$ \\
Hypertension prevalence (no/yes) & $22 / 5$ & $59 / 7$ & $\mathrm{NS}$ \\
HOMA-IR & $3 \pm 0.3$ & $2 \pm 0.13$ & $<0.0001$ \\
Total cholesterol (mmol/l) & $5.79 \pm 0.1$ & $5.25 \pm 0.13$ & 0.03 \\
LDL cholesterol (mmol/l) & $3.7 \pm 0.13$ & $3.39 \pm 0.13$ & $\mathrm{NS}$ \\
\hline
\end{tabular}

NS, not significant.
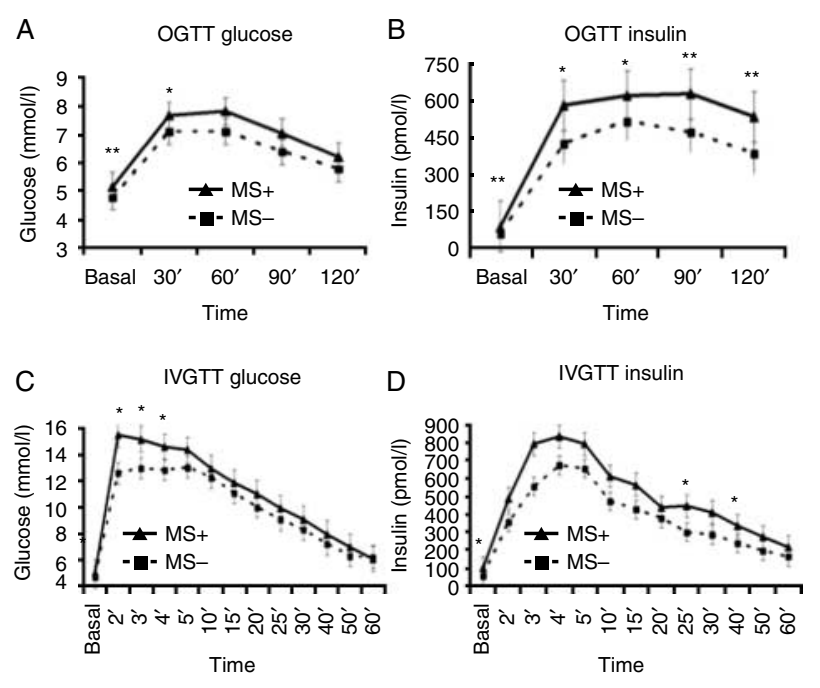

Figure 1 Plasma glucose (panel A) and insulin (panel B) levels during the OGTT and plasma glucose (panel C) and insulin (panel D) levels during the IVGTT, in patients with or without metabolic syndrome. Data are presented as mean \pm S.E.M. ${ }^{\star} P<0.05$;

${ }^{\star \star} P<0.001$.

\section{Plasma glucose, insulin, and C-peptide response during IVGTT}

MS + subjects had elevated plasma glucose levels at baseline, $2^{\prime}(P=0.03), 3^{\prime}(P=0.03)$, and $4^{\prime}(P=0.03$; Fig. 1C). Glucose AUC $(0-10)$ was statistically higher in $\mathrm{MS}+(133.44 \pm 3.89$ vs $118.78 \pm 4.72 \mathrm{mmol} / \mathrm{l} \times \mathrm{min}$, $P=0.04)$, while glucose AUC (0-60) was not statistically different $(576.39 \pm 66.39$ vs $527.17 \pm$ $20.28 \mathrm{mmol} / \mathrm{l} \times \mathrm{min}, P=\mathrm{NS}$ ).

Basal insulin levels were higher in MS + (97.23 \pm 20.83 vs $55.56 \pm 6.94 \mathrm{pmol} / \mathrm{l}, P=0.03$; Fig. 1D). The first phase of insulin release was evaluated by $\triangle$ AIRG. In MS +, it was not significantly different from controls (Table 2). To correct for the higher glucose level in MS + subjects, $\triangle$ AIRG was divided by the incremental glucose peak, $\Delta$ Gp. Similar to $\Delta$ AIRG, $\Delta$ AIRG/ $\Delta$ Gp was also not statistically different (Table 2 ). $\triangle$ AIRG correlated with HOMA-IR $(r=0.4, P<0.05)$.

Furthermore, insulin AUC (0-10) was not significant in both groups, and total insulin AUC (0-60) was significantly higher in MS $+(P=0.04$, Table 2$)$.

$C$-peptide levels were similar in the different time points among the groups as well as AUC (0-10) and AUC (0-60), as shown in Table 2.

\section{Incretin secretion}

Plasma concentrations of GIP and GLP-1 increased significantly after the OGTT.

GIP peaked at $30^{\prime}$ in both groups. MS + individuals showed higher GIP levels at $30^{\prime}(64.2 \pm 9.4$ vs $44 \pm 4.2 \mathrm{pmol} / \mathrm{l}, \quad P=0.03)$, at $60^{\prime}(59.26 \pm 8.04 \mathrm{vs}$ 
Table 2 Main results in normoglucose-tolerant subjects with and without MS. Data are mean \pm S.E.M.

\begin{tabular}{|c|c|c|c|}
\hline & $\mathbf{M S}+(n=29)$ & MS $-(n=67)$ & $P$ value \\
\hline $\mathrm{IG}_{30}(\mathrm{pmol} / \mathrm{mmol})$ & $279 \pm 62.5$ & $155 \pm 17.7$ & 0.01 \\
\hline$\Delta \mathrm{AlR}_{\mathrm{G}}(\mathrm{pmol} / \mathrm{l})$ & $659.7 \pm 111.1$ & $527.8 \pm 69.4$ & NS \\
\hline \\
\hline $\begin{array}{l}\text { (0-120) Insulin OGTT (pmol// min) } \\
\text { Insulin IVGTT (pmol// min) }\end{array}$ & $67838.7 \pm 5875.4$ & $51233.2 \pm 4215.6$ & 0.02 \\
\hline $0-10$ & $6361.62 \pm 944.52$ & $4958.73 \pm 604.21$ & NS \\
\hline $\begin{array}{l}\text { 0-60 } \\
\text { C-peptide IVGTT (nmol// min) }\end{array}$ & $25307.5 \pm 3069.6$ & $18925.1 \pm 2187.6$ & 0.04 \\
\hline $0-10$ & $21.67 \pm 3.66$ & $27.03 \pm 1.86$ & NS \\
\hline $0-60$ & $143.48 \pm 26.3$ & $154.69 \pm 8.65$ & NS \\
\hline \multicolumn{4}{|l|}{ GIP (pmol// min) } \\
\hline $0-30$ & $1248.76 \pm 200.6$ & $831.38 \pm 62.8$ & 0.02 \\
\hline $0-120$ & $6623.96 \pm 871.4$ & $4307.36 \pm 357.4$ & 0.007 \\
\hline \multicolumn{4}{|l|}{ GLP-1 (pmol// min) } \\
\hline $0-30$ & $102.6 \pm 21.4$ & $108.9 \pm 18$ & NS \\
\hline $0-120$ & $400 \pm 68$ & $445 \pm 49$ & NS \\
\hline
\end{tabular}

NS, not significant.

$38.6 \pm 3.6 \mathrm{pmol} / \mathrm{l}, \quad P=0.01)$, at $90^{\prime}(58.4 \pm 7.2$ vs $36.8 \pm 3 \mathrm{pmol} / \mathrm{l}, P=0.003)$, and at $120^{\prime}(58.4 \pm 7.2$ vs $36.4 \pm 3.4 \mathrm{pmol} / \mathrm{l}, P=0.004)$ during the OGTT, as shown in Fig. 2A. Furthermore, AUC (0-30) and AUC (0-120) for GIP were higher in MS + (Table 2).

In contrast to GIP, both GLP-1 kinetic and plasma levels were similar in our experimental groups. In particular, GLP-1 plasma peaks during the OGTT were at $30^{\prime}$ (Fig. 2B). MS + and MS - participants presented no statistically significant differences in GLP-1 plasma levels throughout the entire profile and in the AUC (0-30) and AUC (0-120) for GLP-1 (Table 2).

\section{Glucagon secretion}

During the OGTT, plasma glucagon levels were slightly higher in MS + subjects, although this difference was not statistically significant $(85 \pm 11 \mathrm{vs} 67 \pm 3 \mathrm{ng} / \mathrm{l}, P=0.1)$. In both groups, glucagon levels declined in response to the glucose load (Fig. 2C). However, MS + subjects showed delayed glucagon suppression reaching 28 and $71 \%$ of maximum glucagon suppression respectively, at $30^{\prime}$ and at $60^{\prime}$ of the OGTT in comparison with 81 and $100 \%$ in the MS - group at the same times. During the IVGTT, glucagon responses were similar in the two groups ( $P=$ NS; Fig. 2D).

\section{Discussion}

The main findings of our study are that NGT subjects with MS show an increased GIP secretion and a delayed glucagon suppression after the oral glucose load.

Elevated GIP levels have already been observed in type 2 diabetes (11) and in impaired glucose tolerant patients $(11,12)$, and described as a compensatory mechanism by which these subjects attempt to compensate for their reduced insulinotropic effect of GIP. The reduced insulinotropic response to GIP has been suggested as secondary to a reduction in the $\beta$ cell mass and to impairments in the maximum insulin secretory capacity of the $\beta$ cell (13). In our population, increased GIP levels could represent a compensatory mechanism in response to the increase of plasma glucose observed after the OGTT (AUC 0-120). We could speculate that this continuous stimulation of $\beta$ cells from GIP could lead to an exhaustion of secretory capacity or even induce apoptosis.

The impairment of glucagon suppression that we found in these subjects could represent an immediate price to be paid for this compensatory mechanism. Since GIP has been shown to enhance $\alpha$ cell glucagon
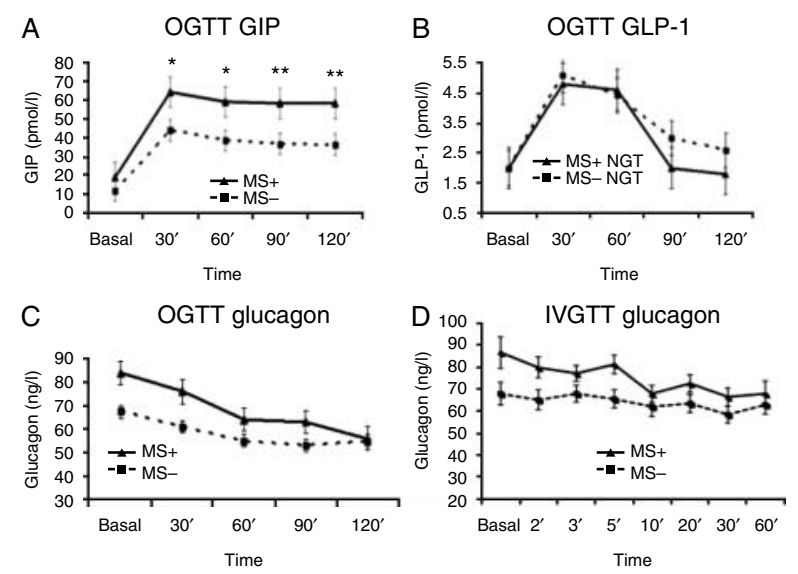

Figure 2 Plasma GIP (panel A), GLP-1 (panel B), glucagon (panel C) levels during the OGTT, and glucagon (panel D) during the IVGTT, in patients with or without MS. Data are presented as mean \pm S.E.M. ${ }^{\star} P<0.05 ;{ }^{\star \star} P<0.001$. 
secretion (5), in contrast with the glucagonostatic GLP1 (4), we can speculate that the increase of GIP could be related to the failure of plasma glucagon concentrations to decrease appropriately after the OGTT. In fact, even if MS + individuals showed no statistically significant differences in plasma glucagon levels at different times, they showed a delayed glucagon suppression reaching 28 and $71 \%$ of maximum glucagon suppression respectively, at $30^{\prime}$ and at $60^{\prime}$ of the OGTT in comparison with 81 and $100 \%$ in the MS - groups at the same times. This event could represent an early defect of $\alpha$ cell function. In contrast to the OGTT, glucagon profiles during the IVGTT were similar between the two groups $(P=N S)$. This finding supports the hypothesis of an incretin axis impairment to explain the $\alpha$ and $\beta$ cell changes observed in subjects with MS. In contrast to GIP, GLP-1 was not significantly different between the two groups. However, in a recent study, subjects with MS had higher fasting GLP-1 levels than those without, and circulating levels of GLP-1 were associated with the number of the MS components (14).

Another finding of our study is that MS + subjects presented, overall, an increased insulin secretion during the OGTT and IVGTT, but different early phases of insulin secretion after the two loads. The insulinogenic index (IG30), an index of early-phase insulin secretion during the OGTT, was higher in MS + subjects. Conversely, during the IVGTT, a well-known test to study the direct effect of glucose on $\beta$ cell function, $\triangle$ AIRG was similar in both study groups. These data, however, should be interpreted with caution because a larger sample size could disclose eventual differences. Similar findings were also obtained when $\triangle$ AIRG was divided by the incremental glucose peak, $\Delta \mathrm{Gp}$, to correct the weight of plasma glucose. The difference of early insulin secretion that we found in response to oral or intravenous glucose, if confirmed, argues against an effect of MS on the $\beta$ cell sensitivity to glucose, and in favor of a direct effect of gut hormones in determining this change.

In a former study, we already showed that subjects affected by MS had a significant increase of early-phase insulin secretion in comparison with subjects without MS, regardless of insulin resistance. This alteration might be a premature feature of the $\beta$ cell dysfunction that eventually leads to overt diabetes (10). Several data suggest that hyperinsulinemia might not only be an adaptive response to insulin resistance, but a primary defect of $\beta$ cell function contributing to glucose intolerance (15).

An impaired incretin effect has been previously reported in other high-risk conditions for diabetes: impaired glucose tolerance (IGT) subjects are characterized by a partial inability of the entero-insular axis to enhance insulin release, which consequently is driven by glucose levels to a greater extent than in normal glucose tolerance (NGT) (16); a reduced insulinotropic effectiveness of GIP was observed in normal glucosetolerant first-degree relatives of patients with type 2 diabetes in comparison with healthy control subjects (17); and a reduced insulinotropic effect of GLP-1 during the hyperglycemic clamp has been observed in risk allele carriers of TCF7L2 rs7903146 (18). In the present study, we did not evaluate the $\beta$ cell responsiveness to incretin infusion. For this reason, we are not able to say if the increased GIP secretion reported in MS subjects could be related to an impaired insulinotropic effect of the above hormone. With regard to incretin secretion, no differences in GLP-1 and GIP response were found in women with a history of gestational diabetes compared with controls (19) either in women with polycystic ovary syndrome (20) or in first-degree relatives of diabetic patients (21).

In conclusion, this study shows that NGT subjects with MS showed increased GIP secretion that could be responsible for the delayed glucagon suppression during the OGTT, thereby suggesting a role for incretins in regulating glucose homeostasis in this condition.

\section{Declaration of interest}

The authors declare that there is no conflict of interest that could be perceived as prejudicing the impartiality of the research reported.

\section{Funding}

This research did not receive any specific grant from any funding agency in the public, commercial or not-for-profit sector.

\section{References}

1 von Lengerke T, Hagenmeyer EG, Gothe H, Schiffhorst G, Happich M \& Haussler B. Excess health care costs of obesity in adults with diabetes mellitus: a claims data analysis. Experimental and Clinical Endocrinology \& Diabetes $2010 \mathbf{1 1 8} 496-504$. (doi:10.1055/s-0030-1253400)

2 Cnop M, Vidal J, Hull RL, Utzschneider KM, Carr DB, Schraw T, Scherer PE, Boyko EJ, Fujimoto WY \& Kahn SE. Progressive loss of beta-cell function leads to worsening glucose tolerance in firstdegree relatives of subjects with type 2 diabetes. Diabetes Care 2007 30 677-682. (doi:10.2337/dc06-1834)

3 Nauck MA, Homberger E, Siegel EG, Allen RC, Eaton RP, Ebert R \& Creutzfeldt $W$. Incretin effects of increasing glucose loads in man calculated from venous insulin and C-peptide responses. Journal of Clinical Endocrinology and Metabolism $1986 \quad 63 \quad 492-498$. (doi:10.1210/jcem-63-2-492)

4 Nauck MA, Kleine N, Orskov C, Holst JJ, Willms B \& Creutzfeldt W. Normalization of fasting hyperglycaemia by exogenous glucagonlike peptide 1 (7-36 amide) in type 2 (non-insulin-dependent) diabetic patients. Diabetologia 199336 741-744. (doi:10.1007/ BF00401145)

5 Meier JJ, Gallwitz B, Siepmann N, Holst JJ, Deacon CF, Schmidt WE \& Nauck MA. Gastric inhibitory polypeptide (GIP) dosedependently stimulates glucagon secretion in healthy human subjects at euglycaemia. Diabetologia $2003 \quad \mathbf{4 6} \quad 798-801$. (doi:10.1007/s00125-003-1103-y)

6 Executive Summary of The Third Report of The National Cholesterol Education Program (NCEP). Expert Panel on Detection, Evaluation, And Treatment of High Blood Cholesterol In Adults (Adult Treatment Panel III). Journal of the American Medical Association 2001285 2486-2497. (doi:10.1001/jama.285.19. 2486) 
7 Report of the expert committee on the diagnosis and classification of diabetes mellitus. Diabetes Care 200326 (Suppl 1) S5-S20. (doi:10.2337/diacare.26.2007.S5)

8 Tura A, Kautzky-Willer A \& Pacini G. Insulinogenic indices from insulin and C-peptide: comparison of beta-cell function from OGTT and IVGTT. Diabetes Research and Clinical Practice $2006 \mathbf{7 2}$ 298-301. (doi:10.1016/j.diabres.2005.10.005)

9 Matsuda M \& DeFronzo RA. Insulin sensitivity indices obtained from oral glucose tolerance testing: comparison with the euglycemic insulin clamp. Diabetes Care 199922 1462-1470. (doi:10.2337/diacare.22.9.1462)

10 Spadaro L, Alagona C, Palermo F, Piro S, Calanna S, Parrinello G, Purrello F \& Rabuazzo AM. Early phase insulin secretion is increased in subjects with normal fasting glucose and metabolic syndrome: a premature feature of beta-cell dysfunction. Nutrition, Metabolism, and Cardiovascular Diseases $201121206-212$. (doi:10.1016/j.numecd.2009.09.003)

11 Vollmer K, Holst JJ, Baller B, Ellrichmann M, Nauck MA, Schmidt WE \& Meier JJ. Predictors of incretin concentrations in subjects with normal, impaired, and diabetic glucose tolerance. Diabetes 200857 678-687. (doi:10.2337/db07-1124)

12 Theodorakis MJ, Carlson O, Muller DC \& Egan JM. Elevated plasma glucose-dependent insulinotropic polypeptide associates with hyperinsulinemia in impaired glucose tolerance. Diabetes Care 200427 1692-1698. (doi:10.2337/diacare.27.7.1692)

13 Meier JJ \& Nauck MA. Is the diminished incretin effect in type 2 diabetes just an epi-phenomenon of impaired beta-cell function? Diabetes 201059 1117-1125. (doi:10.2337/db09-1899)

14 Yamaoka-Tojo M, Tojo T, Takahira N, Matsunaga A, Aoyama N, Masuda T \& Izumi T. Elevated circulating levels of an incretin hormone, glucagon-like peptide-1, are associated with metabolic components in high-risk patients with cardiovascular disease. Cardiovascular Diabetology 20109 17. (doi:10.1186/1475-28409-17)

15 Shanik MH, Xu Y, Skrha J, Dankner R, Zick Y \& Roth J. Insulin resistance and hyperinsulinemia: is hyperinsulinemia the cart or the horse? Diabetes Care 200831 (Suppl 2) S262-S268. (doi:10.2337/dc08-s264)

16 Muscelli E, Mari A, Natali A, Astiarraga BD, Camastra S, Frascerra S, Holst JJ \& Ferrannini E. Impact of incretin hormones on beta-cell function in subjects with normal or impaired glucose tolerance. American Journal of Physiology. Endocrinology and Metabolism 2006291 E1144-E1150. (doi:10. 1152/ajpendo.00571.2005)

17 Meier JJ, Hucking K, Holst JJ, Deacon CF, Schmiegel WH \& Nauck MA. Reduced insulinotropic effect of gastric inhibitory polypeptide in first-degree relatives of patients with type 2 diabetes. Diabetes 200150 2497-2504. (doi:10.2337/diabetes. 50.11.2497)

18 Pilgaard K, Jensen CB, Schou JH, Lyssenko V, Wegner L, Brons C, Vilsboll T, Hansen T, Madsbad S, Holst JJ, Volund A, Poulsen P, Groop L, Pedersen O \& Vaag AA. The T allele of rs7903146 TCF7L2 is associated with impaired insulinotropic action of incretin hormones, reduced $24 \mathrm{~h}$ profiles of plasma insulin and glucagon, and increased hepatic glucose production in young healthy men. Diabetologia 200952 1298-1307. (doi:10.1007/ s00125-009-1307-x)

19 Meier JJ, Gallwitz B, Askenas M, Vollmer K, Deacon CF, Holst JJ, Schmidt WE \& Nauck MA. Secretion of incretin hormones and the insulinotropic effect of gastric inhibitory polypeptide in women with a history of gestational diabetes. Diabetologia 2005 48 1872-1881. (doi:10.1007/s00125-005-1863-7)

20 Svendsen PF, Nilas L, Madsbad S \& Holst JJ. Incretin hormone secretion in women with polycystic ovary syndrome: roles of obesity, insulin sensitivity, and treatment with metformin. Metabolism 200958 586-593. (doi:10.1016/j.metabol.2008. 11.009)

21 Nyholm B, Walker M, Gravholt CH, Shearing PA, Sturis J, Alberti KG, Holst JJ \& Schmitz O. Twenty-four-hour insulin secretion rates, circulating concentrations of fuel substrates and gut incretin hormones in healthy offspring of type II (non-insulin-dependent) diabetic parents: evidence of several aberrations. Diabetologia 199942 1314-1323. (doi:10.1007/ s001250051444)

Received 29 August 2011

Revised version received 1 March 2012

Accepted 5 March 2012 